\title{
A Cross-Layer Heuristic Algorithm for Addressing Shadowing Problem in Optical Attocell Networks
}

\author{
Yuhui $\mathrm{Wu}$ \\ IDLab \\ Department of Information Technology \\ Ghent University-IMEC \\ Ghent, Belgium \\ yuhui.wu@ugent.be
}

\author{
Mario Pickavet \\ IDLab \\ Department of Information Technology \\ Ghent University-IMEC \\ Ghent, Belgium \\ mario.pickavet@ugent.be
}

\author{
Didier Colle \\ IDLab
}

\begin{abstract}
The performance of visible light communication (VLC)-based optical attocell networks degrades due to shadowing caused by opaque objects. In order to improve reliability, we propose a cross-layer algorithm to find the optimal routing and resource allocation schemes when shadowing occurs. Simulation results show that the optimal scheme found by the proposed algorithm significantly improves the performance of optical attocell networks with one randomly shadowed link.

Index Terms-
\end{abstract}

$$
\begin{gathered}
\text { I. Introduction } \\
\eta=\frac{\sum_{u \in \mathbf{U}} \text { end-to-end data rate of } u}{\sum_{u \in \mathbf{U}} \text { requested data rate of } u}
\end{gathered}
$$

$\sum_{u \in \mathbf{U}}$ loads along each route to $u=$ requested rate of $u$

Visible light communication (VLC) has been drawing scientific interests as complement to radio-frequency (RF) wireless technologies. VLC using light-emitting-diodes (LEDs) as transmitters can support simultaneous illumination and wireless access data transmission to user equipment (UE) such as smartphones and laptops. Low-cost photodiodes (PDs) are used as signal receivers by converting radiant intensity to electrical current. Fig. 1(a) illustrates an optical attocell network using LED lamps as base stations (BSs). The backhaul transmission links amongst VLC BSs form a backhaul network. Recently, the interest of using VLC for wireless backhaul transmission has been increasing, as traditional wired backhaul technologies require higher cost for installation and maintenance. Wireless backhaul technologies using line-ofsight (LOS) and mirror-aided non-LOS link configurations as shown in Fig. 1(b) can be easily installed and configured without changing the existing power grid [1], [2]. Both wireless link configurations require auxiliary transceivers. Auxiliary transceivers used in the mirror-aided non-LOS link configuration are oriented toward mirrors or glossy tiles on the floor, while the communicating auxiliary transceivers used in the LOS configuration are directly oriented toward each other. The LOS configuration has more strict alignment requirement to maintain every direct LOS transmission path and therefore more vulnerable to misalignment. The mirror-aided non-LOS link configuration can be an alternative solution when the LOS configuration is not applicable.

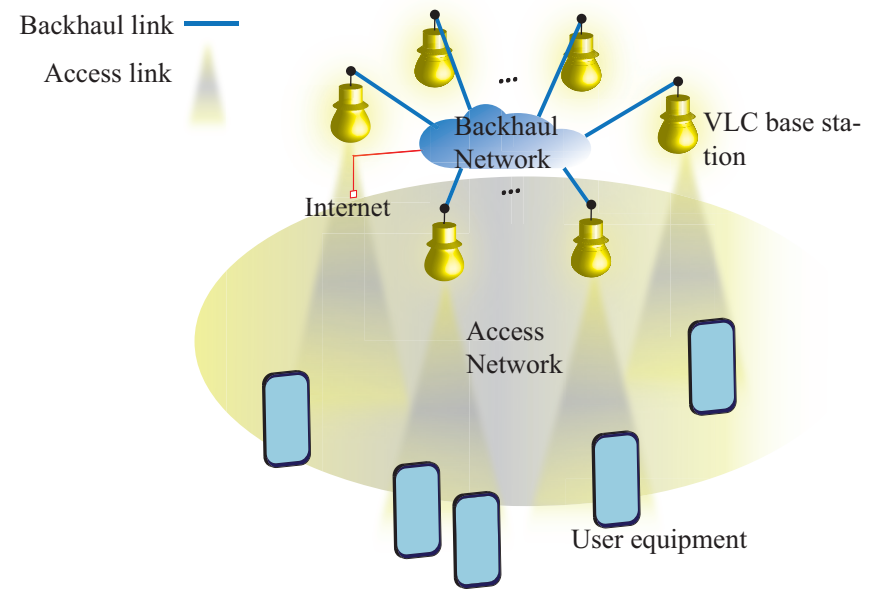

(a)

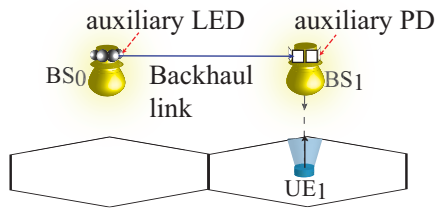

LOS configuration

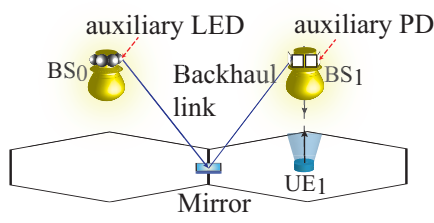

Mirror-aided non-LOS configuration
Fig. 1. (a) An optical attocell network using lamps as base stations; (b) Two wireless backhaul configurations with example backhaul transmission from the gateway $\left(\mathrm{BS}_{0}\right)$ to $\mathrm{BS}_{1}$

One drawback of using VLC for data transmission is that VLC links are vulnerable to shadowing effects caused by interior objects or people. As light rays cannot penetrate opaque objects, blockage of LOS paths or mirror-aided non-LOS paths will cause link failures. Random shadowing due to pedestrian may suddenly degrade network performance especially when using mirror-aided non-LOS backhaul links. As mirror-aided non-LOS backhaul links take advantage of specular reflections from mirrors on the floor, randomly blocked backhaul links 
may prevent transmission to multiple downstream UEs. For many indoor activities such as videoconferencing and teleworking, it is necessary to recover the network from link failures. The network reliability can be improved by network recovery or resilience schemes. A multispot-diffuse multipleinput-multiple-output (MSD-MIMO) scheme was proposed for infrared-based optical wireless networks [4]. Random shadowing could only cause partial obstruction on multispot-diffuse links. The impact of shadowing effects could be minimized by increasing transmitting power on unobstructed links. Authors in [5] proposed a resilience scheme using adaptive equalization and multiple LEDs to send redundant information. Time-division multiple access (TDMA) was used to alleviate inter-symbol interference. The above research only considered physical and multiple access control (MAC) layer recovery schemes. Network layer functions such as routing algorithm and load assignment were not taken into account. In reality, network functions may re-route packets once link blockage is detected. However, routing schemes for optical attocell networks taking into account shadowing effects have not been studied. In addition, inter-cell interference is of importance to the VLC channel performance as both backhaul links and access downlinks use the visible light for transmission. As optical orthogonal frequency division Multiplexing (OFDM) is generally used for VLC, it is straightforward to use orthogonal frequency division multiple access (OFDMA) for channel allocation. The allocation of resource (subcarrier and power) on each subcarrier must be optimized in order to minimize the impact of inter-cell interference and achieve optimal overall performance of the network. We propose a cross-layer resilience scheme to find the optimal routing and resource allocation schemes that improves the network reliability when shadowing occurs. We focus on the single-link failure scenario of the optical attocell network, as measures against all possible link failures are not practical [3]. Simulations of two-tier optical attocell networks with one randomly shadowed link are undertaken. Interference from downlink transmission of activated LEDs in the network is taken into account. Performance of shadowed networks when applying the proposed resilience scheme is shown.

\section{Optical Attocell Networks}

In this article, we consider optical attocell networks using VLC-based wireless backhaul links, i.e. both access and backhaul data are transmitted via VLC links. Mirror-aided nonLOS links are used for backhaul transmission. Each VLC BS uses one LED for access transmission and six auxiliary LEDs for backhaul transmission. OFDMA based on DC-biased optical OFDM (DCO-OFDM) is used.

\section{A. System Model}

Geometry of a two-tier optical attocell network is shown in Fig. 2. BSs are located in cell centers and provide simultaneous illumination and data transmission to UEs. The optical attocell network is a mesh-type network with one gateway and multiple relaying BSs. The gateway BSO is in the center of the network. The first tier $\mathrm{T}_{1}$ consists of neighboring (1hop) BSs of the gateway (BS1 to BS6), and the second tier $\mathrm{T}_{2}$ consists of 2-hop BSs from BS7 to BS18. Every BS has six auxiliary transceivers (auxiliary LEDs and PDs) numbered anti-clockwise as shown in the figure. Each neighboring BS pair communicates with each other via mirror-aided non-LOS backhaul link as shown in Fig. 1(b). The gateway sends downlink data to UEs directly or relayed by neighboring BSs. Access uplinks which normally uses infrared for transmission are not considered in our work. We model the optical attocell network with nodes and links (edges). A set B contains all BS nodes and a set $\mathbf{U}$ contains all UE nodes. A set $\mathbf{L}$ consists of all links in the network. A link $l \in \mathbf{L}$ has a start node $s(l) \in \mathbf{B}$ and an end node $e(l) \in \mathbf{B} \cup \mathbf{U}$. The link $l$ can be either an access link sent from a downward-oriented LED or a backhaul link sent from one of the six auxiliary LEDs. A routing algorithm is performed to choose a route (or multiple routes) for transmitting data from the gateway to UEs. We use a matrix of routing control variables $r_{l}^{u(i)}=\{0,1\}$ to describe the routing policy, where $r_{l}^{u(i)}=1$ means that link $l$ is chosen to send data via the $i$-th route of routes to $u$, and $r_{l}^{u(i)}=0$ otherwise. The number of routes to $u$ is $N_{u}$ which is determined by the routing policy. When there are multiple routes to the same destination, loads allocated to different routes are determined by a load assignment method. Similar to the routing policy, load control variables $a^{u(i)} \leq 1$ are used. The load assigned to the $i$-th route of routes to $u$ is $a^{u(i)} T^{u}$, where $T^{u}$ is the total data rate requested by $u$. The constraint for load assignment is

$$
\sum_{i=1}^{N^{u}} a^{u(i)}=1, \forall u \in \mathbf{U}
$$

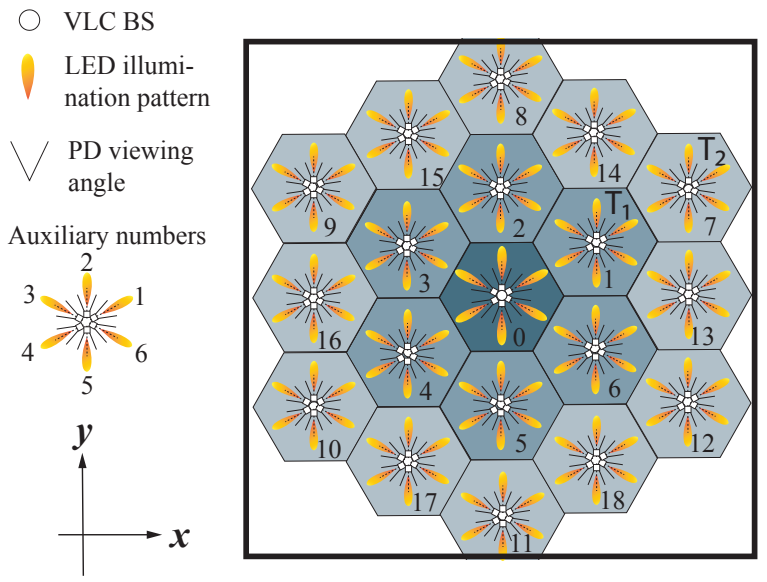

Fig. 2. A two-tier optical attocell network using mirror-aided non-LOS backhaul links

As VLC technology uses intensity modulation, the electrical bandwidth is limited due to the limitation of LEDs' modulation bandwidth. In this work, we consider using blue LEDs with yellow phosphor as VLC transmitters and white-light lumi- 
naires. Each BS is able to use the total available bandwidth $W$. Based on DCO-OFDM, the total bandwidth is divided into $K_{s c}$ subcarriers and $K_{d}$ subcarriers are used for carrying data, where $K_{d}=K_{s c}-2$. In optical OFDM where transmitting signal should be real and non-negative, half of data-carry subcarriers are used to transmit complex conjugate of the other half [6]. Thus, the actual number of subcarriers available to carry data is $K_{d} / 2$. Based on OFDMA, each BS assigns subcarriers to LEDs of all outgoing links based on a bandwidth (subcarrier) allocation method. A control variable $w_{k}^{l}=\{0,1\}$ is used to represent the subcarrier selection policy, where $w_{k}^{l}=1$ means that the $k$-th subcarrier is allocated to link $l$. For each BS, one subcarrier can only be assigned to one link at a time. We have following constraint:

$$
\sum_{\forall l \in\{l: s(l)=b\}} w_{k}^{l} \leq 1, \forall b \in \mathbf{B}
$$

In the case of using white LEDs combining LEDs with multiple wavelengths, for instance, red green blue (RGB) LEDs, wavelength division multiplexing (WDM) and wavelength division multiple access (WDMA) can be used to enable more efficient use of the electrical spectrum. The wavelength selection scheme which is similar to subcarrier selection is not studied in this work, but will be extended in future study.

Due to the illumination and eye safety requirements, optical power transmitted by each LED must be constrained. Downward-oriented LEDs with wide illumination pattern are generally used for illumination. Optical power from auxiliary LEDs which are more directed and concentrated should be limited to ensure eye safety. We assume that all downwardoriented LEDs transmit the same optical power $p_{o p t}^{a}$ and set the transmitted optical power of each auxiliary LED as $0.1 p_{o p t}^{a}$. A power allocation scheme determines the amount of power allocated to each subcarrier and a constraint

$$
\sum_{k=1}^{K_{s c}} p_{k}^{l} \leq p_{\text {elec }}^{l}, \forall l \in \mathbf{L}
$$

should be met, where $p_{k}^{l}$ is the electrical power allocated on the $k$-th subcarrier of link $l$ and $p_{\text {elec }}^{l}$ is the total electrical power available for a LED transmitter of link $l$. The electrical power $p_{\text {elec }}^{l}$ is calculated by a relationship $\alpha=\frac{p_{\text {opt }}^{l}}{\sqrt{p_{\text {elec }}^{l}}}$, where $\alpha$ is the DC-bias factor [6].

\section{B. Performance Metrics}

The performance of the network is assessed by a successful transmission ratio

$$
\eta=\frac{\sum_{u \in \mathbf{U}} \hat{T}^{u}}{\sum_{u \in \mathbf{U}} T^{u}}
$$

where $T^{u}$ is the data rate requested by $u$ and $\hat{T}^{u}$ is the actual end-to-end data rate to $u$. The actual end-to-end data rate is calculated by

$$
\hat{T}^{u}=\sum_{i=1}^{N_{u}} \min \left(a^{u(i)} T^{u}, \min _{\forall l \in \mathbf{L}} C_{l}^{u(i)}\right)
$$

where $C_{l}^{u(i)}$ is the portion of the capacity of link $l$ available for the $i$-th route to $u$. The capacity portion $C_{l}^{u(i)}$ is calculated based on the requested load:

$$
C_{l}^{u(i)}=\frac{r_{l}^{u(i)} a^{u(i)} T^{u}}{\sum_{\forall \mu \text { in } \mathbf{U}} \sum_{j=1}^{N_{\mu}} r_{l}^{\mu(j)} a^{\mu(j)} T^{\mu}} C_{l}
$$

where $C_{l}$ is the capacity of link $l$. Link capacity can be calculated using Shannon's theorem $C_{l}=\sum_{k=1}^{K_{s c}} W_{k} \log _{2}\left(1+\gamma_{k}^{l}\right)$, where $W_{k}=W / K_{s c}$ is the bandwidth of each subcarrier, and $\gamma_{k}^{l}=\frac{w_{k}^{l} p_{k}^{l}\left|G_{k}^{l}\right|^{2}}{\sigma_{k}^{2}+I_{k}^{l}}$ is the signal-to-interference-plus-noise ratio (SINR) on the $k$-th subcarrier, $G_{k}^{l}$ is the channel gain of the $k$-th subcarrier of link $l, \sigma_{k}^{2}=N_{0} W / K_{s c}$ is the noise power on the $k$-th subcarrier and $N_{0}$ is the power spectral density of the additive white Gaussian noise. $I_{k}^{l}$ is the total interference power on the $k$-th subcarrier of link $l$, which is the sum of power received by the end node of $l$, i.e. $I_{k}^{l}=\sum_{m \in\{m: e(m) !=e(l), \forall m \in \mathbf{L}\}} w_{k}^{m} p_{k}^{m}\left|G_{k}^{m}\right|^{2}$. The SINR calculation is taking into account the bit-error rate requirement $B E R=10^{-6}$. The channel gain $\left|G_{k}^{l}\right|$ of LOS links and mirror-aided non-LOS links can be calculated based on transceivers' locations and orientations, which has been well studied [1], [7]. To simplify the calculation, only the dominant LOS path in access downlink and the dominant first-bounce specular reflection in backhaul links are considered in our simulations. Multi-path effect caused by multi-bounce diffuse reflections is neglected and the channel gain is considered flat across the electrical spectrum.

\section{RESILIENCE SCHEME}

As light rays cannot penetrate opaque objects, the performance of VLC transmission links which utilize the dominant LOS (or the first-bounce specular reflection) component will be degraded when shadowing occurs. We assume that a link is completely blocked by pedestrians and the transmission of the shadowed link is totally broken. Once the shadowing occurs, the capacity of the obstructed link drops to zero and network performance metric $\eta$ decreases due to the reduction of $\sum_{u \in \mathbf{U}} \hat{T}^{u}$. From the previous section, it is known that $\hat{T}^{u}$ changes with control variables $r_{l}^{u(i)}, a^{u(i)}, w_{k}^{l}$, and $p_{k}^{l}$. In order to guarantee the network reliability, control policies (routing, load assignment, subcarrier selection and power allocation) must be optimized such that $\hat{T}^{u}$ is maximized by choosing proper control variables. The problem is stated as below:

maximize (4)

subject to (1), (2), (3)

We propose a cross-layer algorithm RASP to address the problem. The algorithm chooses different combinations of control options denoted by $(\mathrm{R}, \mathrm{A}, \mathrm{S}, \mathrm{P})$ and calculates the objective value $\eta$. There could be a large number of control options that can be applied in RASP. It is not practical to investigate all control options. Thus, the (sub)optimal combination of given control options is heuristically searched by running RASP in optical attocell networks with one randomly shadowed link. 
The control options considered in the heuristic method are described in this section. A central controller, which can be a software-defined-network (SDN) controller, sends the chosen combination of control options to BSs in the optical attocell network.

\section{A. Routing Policy Option (R)}

Before starting the routing algorithm, each UE is assigned to the nearest BS. There exists an directional edge with cost $=1$ from a BS to a UE who is assigned to it. Each neighboring BS-pair is connected by bi-directional edges with cost $=1$.

1) Shortest Path Routing: $\mathrm{R}=1$

If the shortest path routing is chosen, Dijkstra routing algorithm is applied to find the path with minimum hopcount from the gateway to each UE. If there are multiple shortest paths, the routing algorithm randomly chooses one, i.e. $\max \left(N_{u}\right)=1, \forall u \in \mathbf{U}$.

2) K-Shortest Path Routing: $\mathrm{R}>1$

If the traffic is congested in some links, it is better to choose longer paths with light-loaded or unused links. As traditional transmission control protocol (TCP) can use packet number to reorder the incoming packets, it is possible to send packets via paths with different hops. With this option, K-shortest path routing algorithm is performed. If routes to some UEs contain overloaded links, Yen's K-shortest path routing algorithm will be used to discover the first $K$ shortest paths to those UEs. Here, the value of $K$ is the value of control option R.

\section{B. Load Assignment Policy Option (A)}

1) Fair Assignment: $\mathrm{A}=1$

The simplest load assignment method is to evenly distribute data amongst all paths to the UE, i.e. $a^{u(i)}=1 / N_{u}$.

2) Weighted-Fair Assignment: $\mathrm{A}=2$

As link capacities are limited, the fair assignment may not be suitable if too many loads are assigned on some bottleneck links. If there are more than one route to a user $u$, i.e. $N_{u}>1$, we allocate the load based on weights of links along each route. The weight of a link $l$ which is denoted by $f_{l}$ is the reciprocal of the total number of routes with $r_{l}^{\mu(i)}=1, \mu \in \mathbf{U}$, such that the more routes going through a link, the lower the weight. The weight of a route $f^{u(i)}$ is the minimum value of $f_{l}$ of links along this route. The load assignment control variable is $a^{u(i)}=\frac{f^{u(i)}}{\sum_{i=0}^{N_{u}^{-1}} f^{u(i)}}$.

\section{Subcarrier Allocation Policy (S)}

Based on the load assignment method, the number of subcarriers allocated to each link can be determined. The number of subcarriers required by each link can be estimated by the sum of loads on this link $T_{l}=\sum_{u \in \mathbf{U}} \sum_{i=0}^{N_{u}-1} r_{l}^{u(i)} a^{u(i)} T^{u}$. The actual number of subcarriers is limited by the number of available subcarriers $K_{d} / 2$ of a BS. For each BS $b \in \mathbf{B}$, the number of subcarriers allocated to link $l$ with $e(l)=b$ is

$$
k_{l}=\frac{T_{l}}{\sum_{m \in\{m: e(m)=b\}} T_{m}} \frac{K_{d}}{2}
$$

1) Cyclic Subcarrier Allocation: $\mathrm{S}=1$

As shown in Fig. 2, LEDs are numbered according to their orientations. We allocate subcarriers to links in an cyclic order of their transmitting LEDs.

2) Random Subcarrier Allocation: $\mathrm{S}=2$

With this option, we randomly allocate subcarriers based on the calculated number of subcarriers for each link. This option can be done multiple times to find the optimal allocation.

\section{Power Allocation Policy (P)}

For all $N_{d} / 2$ subcarriers carrying the real data, half of the electrical power should be allocated on them. The other half electrical power is allocated to their complex conjugates. Before applying following power allocation policy, the central controller equally allocates total transmitting power to all subcarriers carrying the real data, i.e. $p_{k}^{l}=p_{\text {elec }}^{l} / N_{d}$. The controller then calculates the SINR, major interfering source of each subcarrier of each link, and the actual link capacity based on current control policies.

\section{1) Adaptive Power Allocation: $\mathrm{P}=1$}

If one or more links are overloaded, i.e. the actual link capacity is less than the assigned load, the power is re-allocated based on the major interfering source information. We generate a matrix of risky tags $r t_{s, r, k}$, where the transmitter of link $s$ is a possible interfering source of the receiver of link $r$, and $k$ is the index of the subcarrier. If $s$ is the major interfering source of $r$ on the $k$-th subcarrier, i.e. the transmitter of link $s$ causes the largest interference to the receiver of the link $r$ on the $k$-th subcarrier, $r t_{s, r, k}$ is set to be 1 , and 0 otherwise. Reallocating power on subcarriers of a link $s$ with $r t_{s, r, k}=0$ has less impact on the overall performance. Water-filling algorithm is used to re-allocate power that maximizes the link capacity taking into account the total power constraint [8]. The total amount of power is the sum of previously allocated power on subcarriers with $r t_{s, r, k}=0$. When applying the water-filling algorithm, current SINR information is used.

\section{2) Adaptive Power Allocation with Relaxation: $\mathrm{P}=2$}

The difference between two adaptive power allocation options $\mathrm{P}=1$ and $\mathrm{P}=2$ are the number of subcarriers that need to be reallocated. Based on the link capacities and load assignment information, a matrix of relax tags $x t_{s, r, k}$ is generated to denote the relaxation of a link. $x t_{s, r, k}=1$ only if $r t_{s, r, k}=1$ and the ratio between the capacity of $s$ and the load assigned to $s$ is larger than a predefined relaxing ratio $\epsilon$. In addition to power on subcarriers with $r t_{s, r, k}=0$, power on subcarriers with $x t_{s, r, k}=1$ are also reallocated using waterfilling algorithm.

\section{E. RASP Algorithm and Heuristic}

When a network is established, the central controller collects the information of network topology and channel states. Then, it calculates the successful transmission ratio $\eta$ of shadowed networks with different throughput requirements when applying RASP algorithm. The optimal combination of options is the one giving the maximum $\eta$ with the most times. This heuristically found optimal combination of control options 
is applied in the given network whenever link blockage is detected.

The pseudo-code of RASP algorithm is shown below. The function obj(R,A,S,P) is the calculation using (6) by taking control variables determined by the combination of control options (R,A,S,P). As some options require the information of current network status, for instance, $\mathrm{R}>1$ option requires overload information, every calculation of $\operatorname{obj}(\mathrm{R}, \mathrm{A}, \mathrm{S}, \mathrm{P})$ is initialized with the option $(\mathrm{R}, \mathrm{A}, \mathrm{S}, \mathrm{P})=(1,1,1,1) . \mathrm{R}_{\max }$ is the maximum number of routes discovered by $\mathrm{k}$-shortest paths routing. $S_{\max }$ is the number of attempts for random subcarrier allocation and $S_{\max }=1$ if $\mathrm{S}=1$.

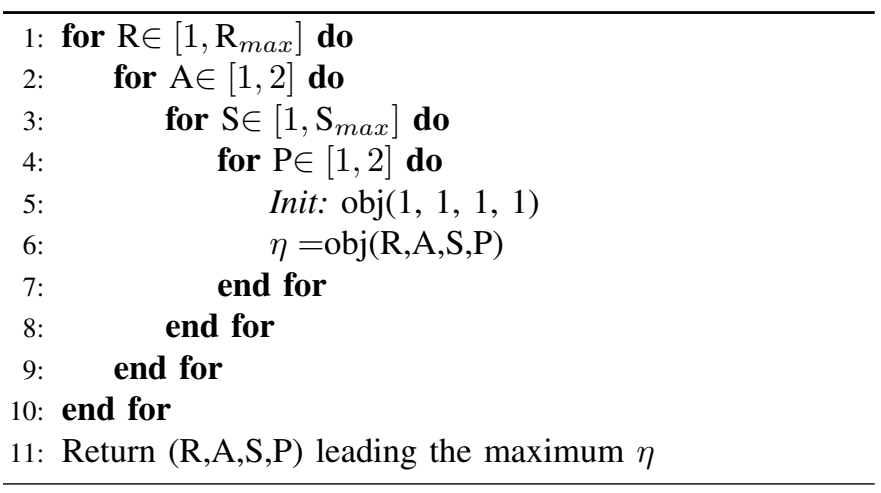

\section{Simulation And Results}

We implemented RASP and the heuristic method in Python. The proposed resilience scheme based on RASP and heuristic was simulated with two-tier optical attocell networks using mirror-aided non-LOS backhaul links. In the first step of simulation, we heuristically searched the optimal combination of control options (R,A,S,P) in two-tier networks as shown in 2. We generated two-tier networks with different user densities and requested data rates. The UE distribution followed Poisson Point Process (PPP) with user density $\lambda$. Requested data rates by UEs were randomly chosen from a set $\{0.032 \mathrm{Mb} / \mathrm{s}, 0.064$ $\mathrm{Mb} / \mathrm{s}, 0.256 \mathrm{Mb} / \mathrm{s}, 0.4 \mathrm{Mb} / \mathrm{s}, 1 \mathrm{Mb} / \mathrm{s}\}$, which corresponds to data rates of most indoor activities such as Internet access and voice over IP. The requested data rate for each UE was constant during the simulation. The shadowing effect was modeled as random deactivation of one active link (an access link or a backhaul link which had been activated for data transmission). Network related parameters are set as following: $W=40 \mathrm{MHz} ; p_{o p t}^{a}=10 \mathrm{~W}$; DC-bias factor $\alpha=3$; $N_{0}=1 \times 10^{-21} \mathrm{~A}^{2} / \mathrm{Hz}$; cell radius is $2 \mathrm{~m}$; heights of BSs and UEs are $3 \mathrm{~m}$ and $1 \mathrm{~m}$, respectively; the reflectively of mirrors (for backhaul transmission) 0.9 ; the responsivity of PDs is $0.5 \mathrm{~A} / \mathrm{W}$. The relaxing ratio of $\mathrm{P}=2$ option is set to be $\epsilon=2$. We set the maximum iteration of $\mathrm{R}$ and $\mathrm{S}$ as $\mathrm{R}_{\max }=13, \mathrm{~S}_{\max }=5$. We ran the heuristic method in shadowed two-tier networks with user density $\lambda$ from $0.01 \mathrm{user} / \mathrm{m}^{2}$ ( 0.13 user/attocell) to $0.1 \mathrm{user} / \mathrm{m}^{2}$ (1.3 user/attocell). The optimal combination found by running 500 simulations was $(\mathrm{R}, \mathrm{A}, \mathrm{S}, \mathrm{P})=(11,2,2,2)$.
In the second step of simulation, we investigated the performance of the resilience scheme. The resilience scheme with $(\mathrm{R}, \mathrm{A}, \mathrm{S}, \mathrm{P})=(11,2,2,2)$ was simulated in more than 500 networks and average values of $\eta$ were calculated. The performance of unshadowed networks, shadowed networks, and shadowed networks recovered by using the heuristically found optimal combination were compared. For comparison, the unshadowed scenario also applied the same control options as the recovery scheme. The performance of network using the resilience scheme is shown in Fig. 3. The unshadowed performance is 1.0 when $\lambda \leq 0.06$. For networks with higher user density, the data requests may exceed the network capacity, which is limited by the electrical bandwidth. It can be seen that network performance dramatically degrades due to shadowing effects. When user density is low, the blockage of one active transmission link has large impact on the overall network performance. More then $50 \%$ of data rate requests cannot be fulfilled when $\lambda=0.01$. This is likely due to the fact that the amount of UEs is very low when $\lambda=0.01$ (mostly with one or two UEs in the network). The shadowed link might have been used for transmitting a large fraction of total requested data rates. Thus, the failure of one of this type of links dramatically drops $\hat{T}^{u}$ for one or more UEs. The performance degradation can be addressed by applying the resilience scheme. As shown in Fig. 3, the network performance significantly improves when recovered by applying the optimal combination of options. The recovered performance is closed to the network performance without shadowing effects. The performance enhancement is significant in low user density scenario. All data rate requests can be fulfilled after recovery when $\lambda \leq 0.05$. The network successful transmission ratio $\eta$ of recovered shadowed networks is less than that of unshadowed networks with high user densities. This is likely due to the limited number of control options investigated with the heuristic method. Better performance could be achieved when applying more control options at the expense of time.

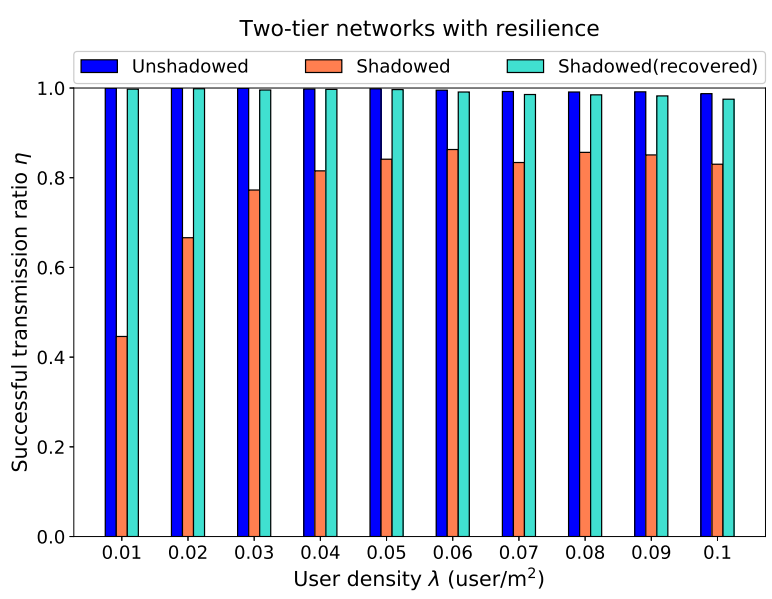

Fig. 3. Successful transmission ratio $\eta$ vs. user density $\lambda$ of two-tier networks without shadowing, with shadowing, and recovered from shadowing effects 


\section{CONCLUSIONS}

A resilience scheme for addressing shadowing problems in optical attocell networks is proposed. By heuristically running the cross-layer algorithm RASP, which combines four control policies (routing policy, load assignment, bandwidth allocation and power allocation), the optimal combination of control options for the given optical attocell network is found. The corresponding control policies are sent to VLC BSs in the network by a central controller. Simulations of two-tier optical attocell networks with one randomly shadowed link are undertaken. It is shown that the recovered performance is closed to the network performance without shadowing effects especially when user density is low. Future work includes designing and implementing of protocols using the proposed algorithm.

\section{REFERENCES}

[1] Y. Wu, P. Audenaert, M. Pickavet, and D. Colle, "Mirror-aided non-LOS VLC channel characterizations with a time-efficient simulation model", Photonic Network Communications, vol. 38, pp. 151-166, 2019

[2] H. Kazemi, M. Safari, and H. Haas, "A wireless optical backhaul solution for optical attocell networks", IEEE Transactions on Wireless Communications, vol. 18, pp. 807-823, 2019

[3] J. P. Vasseur, M. Pickavet, and P. Demeester, Network Recovery, 2nd ed., Morgan Kaufmann, 2004

[4] S. Jivkova, B. A. Hristov, and M. Kavehrad, "Power-efficient multispotdiffuse to broad-band optical wireless communications", IEEE Transactions on Vehicular Technology, vol. 53, pp. 882-889, 2004

[5] T. Komine, J. H. Lee, S. Haruyama, and M. Nakagawa, "Adaptive equalization system for visible light wireless communication utilizing multiple white led lighting equipment", IEEE Transactions on Wireless Communications, vol. 8, pp. 2892-2900, 2009

[6] J. Armstrong and B. J. C. Schmidt, "Comparison of asymmetrically clipped optical OFDM and DC-biased optical OFDM in AWGN", IEEE Communications Letters, vol. 12, pp. 343-345, 2008

[7] J. R. Barry, J. M. Kahn, W. J. Krause, E. A. Lee, and D. G. Messerschmitt, "Simulation of multipath impulse response for indoor wireless optical channels", IEEE Journal on Selected Areas in Communications, vol. 11 , pp. 367-379, 1993

[8] A. F. Molish, "Wireless Communications", 2nd ed., John Wiley and Sons, 2011 